\title{
Recent Advances in Real-Time Musical Effects, Synthesis, and Virtual Analog Models
}

\author{
Jyri Pakarinen, ${ }^{1}$ Vesa Välimäki, ${ }^{1}$ Federico Fontana, ${ }^{2}$ \\ Victor Lazzarini, ${ }^{3}$ and Jonathan S. Abel ${ }^{4}$ \\ ${ }^{1}$ Department of Signal Processing and Acoustics, Aalto University School of Electrical Engineering, 02150 Espoo, Finland \\ ${ }^{2}$ Department of Mathematics and Computer Science, University of Udine, 33100 Udine, Italy \\ ${ }^{3}$ Sound and Music Technology Research Group, National University of Ireland, Maynooth, Ireland \\ ${ }^{4}$ CCRMA, Stanford University, Stanford, CA 94305-8180, USA
}

Correspondence should be addressed to Jyri Pakarinen, jyri.pakarinen@tkk.fi

Received 8 October 2010; Accepted 5 February 2011

Academic Editor: Mark Kahrs

Copyright (C) 2011 Jyri Pakarinen et al. This is an open access article distributed under the Creative Commons Attribution License, which permits unrestricted use, distribution, and reproduction in any medium, provided the original work is properly cited.

This paper reviews some of the recent advances in real-time musical effects processing and synthesis. The main emphasis is on virtual analog modeling, specifically digital emulation of vintage delay and reverberation effects, tube amplifiers, and voltagecontrolled filters. Additionally, adaptive effects algorithms and sound synthesis and processing languages are discussed.

\section{Introduction}

Real-time musical effects processing and synthesis play a part in nearly all musical sounds encountered in the contemporary environment. Virtually all recorded or electrically amplified music in the last few decades uses effects processing, such as artificial reverberation or dynamic compression, and synthetic instrument sounds play an increasingly larger part in the total musical spectrum. Furthermore, the vast majority of these effects are presently implemented using digital signal processing (DSP), mainly due to the flexibility and low cost of modern digital devices. For live music, realtime operation of these effects and synthesis algorithms is obviously of paramount importance. However, also recorded music typically requires real-time operation of these devices and algorithms, because performers usually wish to hear the final, processed sound of their instrument while playing.

The purpose of this article is to provide the reader with an overview of some of the recent advances in this fascinating and commercially active topic. An exhaustive review of all novel real-time musical effects processing and synthesis would fill a book. In fact, an earlier review on digital audio effects can be found in the book [1] and in a recent book [2], while reviews of virtual analog modeling and digital sound synthesis can be found in articles [3] and [4], respectively. A tutorial on virtual analog oscillator algorithms, which are not tackled in this paper, has been written by Välimäki and Huovilainen [5]. Also, musical synthesis and effects applications for mobile devices have been reported in [6]. In order to conveniently fit in a single journal article, a selection of some of the most active subtopics under this exciting research field have been chosen for presentation here.

The organization of this review is as follows: adaptive effects processing algorithms, such as the adaptive FM technique, are reviewed in Section 2. Section 3 discusses the emulation of vintage delay and reverberation effects, while recent advances in tube amplifier emulation are studied in Section 4. Real-time simulation of an interesting analog effects device, the voltage-controlled filter, is reviewed in Section 5, and recent advances in sound synthesis and processing languages are discussed in Section 6. Finally, Section 7 concludes the review.

\section{Adaptive Effects Processing}

Many adaptive effects processing algorithms suitable for a general input signal have been introduced during the past few 


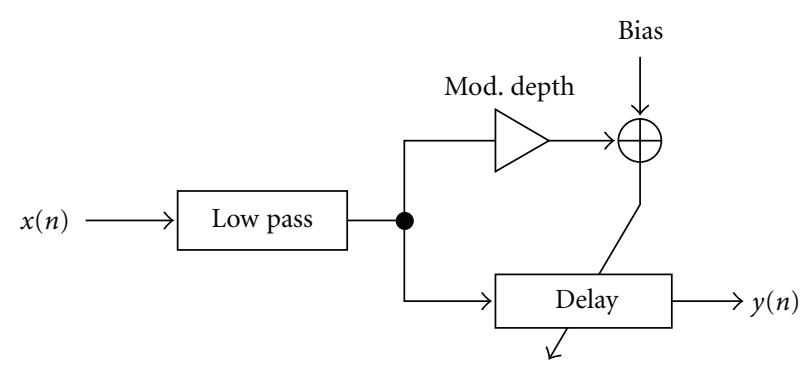

(a)

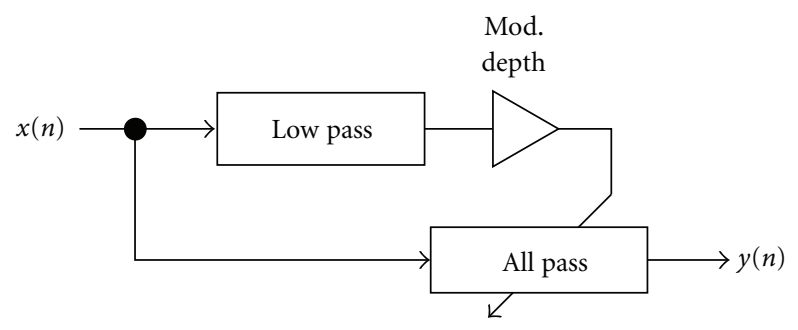

(c)

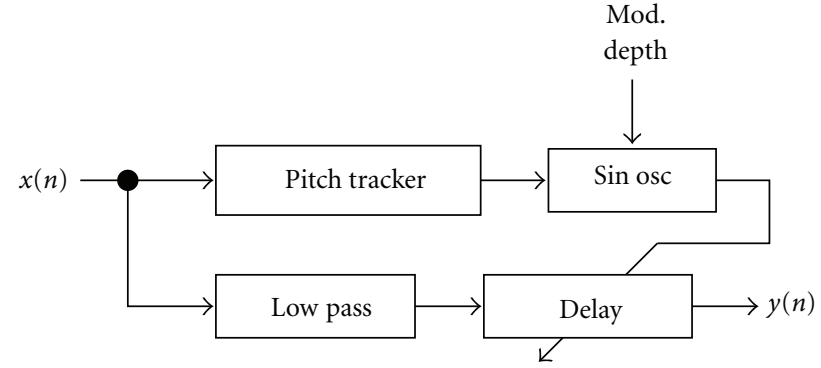

(b)

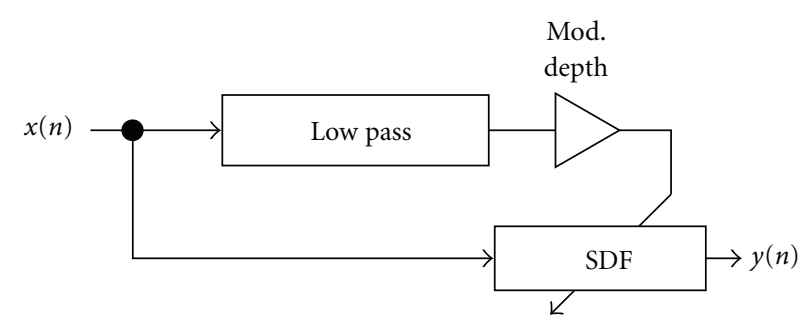

(d)

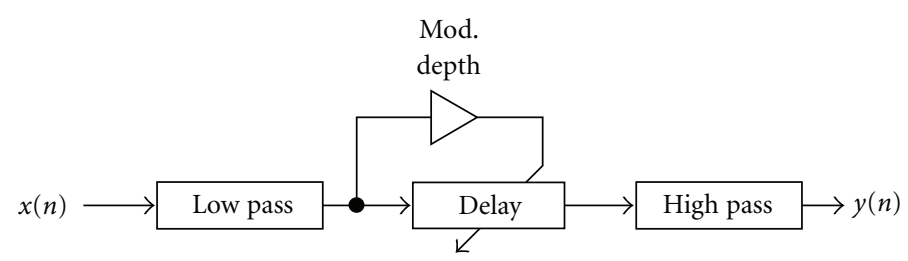

(e)

FIGURE 1: Recent adaptive effects processing structures: (a) self-modulating FM [7], (b) adaptive FM [8], (c) coefficient-modulated all-pass filter [9], (d) coefficient-modulated spectral delay filter (SDF) [10], and (e) brassification [11].

years. The idea of an adaptive audio effect is not entirely new: it has been possible for many years to control parameters of an algorithm with a feature measured from the signal. Still, it was found useful to give the name "Adaptive DAFx" to this class of methods a few years ago [12], and since then many papers belonging to this category have been published. In this section, we briefly review some recent methods belonging to this category of real-time musical signal processing algorithms.

Audio-driven sound synthesis introduced by Poepel and Dannenberg [7] is an example of a class of adaptive effects, which goes so far as almost being a synthesis method rather than a transformation of the input signal. In one example application of this idea, Poepel and Dannenberg show how FM (frequency modulation) synthesis can be modified by deriving the modulation signal frequency by tracking the pitch of an input signal. In this case, the input signal is assumed to be a monophonic signal, such as a trumpet sound picked up by a microphone. Poepel and Dannenberg also describe an algorithm, which they call self-modulating FM. In this method, the low-pass filtered input signal is used as both modulation and carrier signal. The modulation is realized by varying the delay-line length with the scaled lowpass filtered input signal, see Figure 1(a) [13].

Lazzarini and his colleagues [8] extended the basic idea of audio-driven synthesis to what they call adaptive FM
(AdFM). Poepel and Dannenberg had proposed a basic modified FM synthesis method in which the modulator is replaced with the input audio signal [7]. Lazzarini et al. [8] reversed the roles of the modulator and the carrier so that they use the input signal as the carrier. It is advantageous to low-pass filter the carrier signal before modulating it, since the spectrum of the signal will expand because of frequency modulation and the output sound will otherwise become very bright. The pitch of the input signal, however, is used to control the modulation frequency. In AdFM, the modulation is implemented by moving the output tap of a delay line at the modulation frequency, as shown in Figure 1(b). A fractional delay filter is required to obtain smooth delay variation [14]. The FM modulation index then controls the width of this variation. An advantage of the AdFM effect is that it retains the character of the input signal. In one extreme, when the modulation depth is set to zero, the output signal will be identical to the (low-pass filtered) input signal. By increasing the modulation index, the method distorts the input signal so that it sounds much like an FMsynthesized tone.

Extensions to these methods were presented in [15], where the FM sidebands were split in four separate groups (in combinations of upper/lower and even/odd), and in [16] where asymmetric-spectra FM methods were introduced. Finally, in [17] a modified FM version was presented 


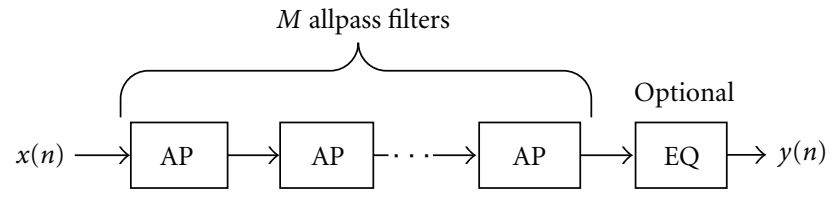

Figure 2: A spectral delay filter consists of a cascade of all-pass filters (AP) and an optional equalizing filter (EQ) [18].

(a variant of FM based on modified Bessel coefficients). This was complemented by an algorithm that allows transitions between modified, asymmetrical, and classic FM for adaptive applications.

An adaptive effect of a similar spirit as the audio-driven approach and adaptive FM was introduced by Pekonen [9]. In his method, presented in Figure 1(c), the audio signal is filtered with a first-order all-pass filter and the coefficient of that all-pass filter is simultaneously varied with scaled and possibly low-pass filtered version of the same input signal. This technique can be seen as signal-dependent phase modulation and it introduces a distortion effect, but does not require a table lookup, like waveshaping, or pitch tracking, like AdFM.

It was shown recently by Lazzarini et al. [19] that the choice of the all-pass filter structure affects considerably the output signal in the time-varying case. It was found that the direct form I structure has smaller transients with the same input and coefficient modulation signals than two alternative structures and, thus, this expression is recommended for use in the future

$$
y(n)=x(n-1)-a[x(n)-y(n-1)],
$$

where $x(n)$ and $y(n)$ are, respectively, the input and output signals of the all-pass filter and $a$ is the all-pass filter coefficient.

Kleimola and his colleagues [10] combined and expanded further the idea of the signal-adaptive modulation utilizing all-pass filters. In this coefficient-modulated method, the input signal is fed through a chain of many identical first-order all-pass filters while the coefficients are modulated at the fundamental frequency of the input signal. The chain of all-pass filters cascaded with an optional equalizing filter, as shown in Figure 2, is called a spectral delay filter [18]. A pitch tracking algorithm or low-pass filtered input signal may be used as a modulator, see Figure $1(\mathrm{~d})$. The modulation of the common all-pass filter coefficient introduces simultaneously frequency and amplitude modulation effects [10].

The "brassifier" effect proposed by Cooper and Abel [11] is another new technique that is closely related to the previous ones. It has been derived from the nonlinear acoustic effect that takes place inside brass musical instruments, when the sound pressure becomes very large. In the "brassification" algorithm, the input signal is scaled and is used to control a fractional delay, which phase modulates the same input signal. It can be seen that the brassification method differs from the self-modulating FM method of Figure 1(a) in its computation of the delay modulation and in that a highpass filter is used as postprocessing. Similar methods have previously been used in waveguide synthesis models to obtain interesting acoustic-like effects, such as generic amplitude-dependent nonlinear distortion [20], shock waves in brass instruments [21-23], and tension-modulation in string instruments $[24,25]$. These methods aim at implementing a passive nonlinearity [26]. All these nonlinear effects are implemented by controlling the fractional delay with values of the signal samples contained in the delay line.

In the practical implementation of the brassification method, the input signal propagates in a long delay line and the output is read with an FIR interpolation filter, such as linear interpolation or fourth-order Lagrange interpolation. The input signal can be optionally low-pass filtered prior to the delay-line input to emphasize its low-frequency content and the output signal of the delay line can be high-pass filtered to compensate the low-pass filtering, as shown in Figure 1(e).

\section{Vintage Delay and Reverberation Effects Processor Emulation}

Digital emulation of vintage electronic and electromechanical effects processors has received a lot of attention recently. While their controls and sonics are very desirable, and the convenience of a software implementation of benefit, these processors often present signal processing challenges making real-time implementation difficult. In this section, we focus on recent signal processing techniques for realtime implementation of vintage delay and reverberation effects. We first consider techniques to emulate reverberation chambers, and spring and plate reverberators, and then focus on tape delay, bucket brigade delays, and the Leslie speaker.

3.1. Efficient Low-Latency Convolution. Bill Putnam, Sr. is credited with introducing artificial reverberation to recording [27]. The method involved placing a loudspeaker and microphone in a specially constructed reverberation chamber made of acoustically reflective material and having a shape lacking parallel surfaces. The system is essentially linear and time invariant and, therefore, characterized by its impulse response. Convolving the input signal with the chamber impulse response is a natural choice, as the synthesized system response will be psychoacoustically identical to that of the measured space.

However, typical room impulse responses have long decay times and a real-time implementation cannot afford the latency incurred using standard overlap-add processing [28]. Gardner [29] and McGrath [30] noted that if the impulse responses were divided into two sections, the computation would be nearly doubled, but the latency would be halved. Accordingly, if the impulse response head is recursively divided in two so that the impulse response section lengths were $[L, L, 2 L, 4 L, 8 L, \ldots]$, the initial part of the impulse response would provide the desired low latency, while the longer blocks comprising the latter portion of the impulse response would be efficiently computed. 
Garcia in [31] noted that processors could efficiently implement the needed multiply-accumulate operations, but that the addressing involved slowed FFT operations for longer block sizes. If a number of blocks were of the same length, then they could all share the input signal block forward transform. For example, if the impulse responses were divided into sections of identical block lengths, only one forward transform and only one inverse transform would be needed for each block of input signal block processed. Garcia showed that dividing the impulse response into a few sections, each of which is divided into equal-length blocks, produces great computational savings while maintaining a low latency.

Finally, it should be pointed out that an efficient method for performing a low-latency convolution, dividing the impulse response into equal-length blocks and using a twodimensional FFT, was introduced by Hurchalla in [32].

3.2. Hybrid Reverberation Methods. Reverberation impulse responses contain a set of early arrivals, often including a direct path, followed by a noise-like late field. The late field is characterized by Gaussian noise having an evolving spectrum $P(\omega, t)[33,34]$

$$
P(\omega, t)=|q(\omega)|^{2} \exp \left\{-\frac{t}{\tau(\omega)}\right\},
$$

where the square magnitude $|q(\omega)|^{2}$ being the equalization at time $t=0$, and $\tau(\omega)$ defining the decay rate as a function of frequency $\omega$. This late field is reproduced by the feedback delay network (FDN) structure introduced by Jot in the early 1990s [34]. There, a signal is delayed by a set of delay lines of incommensurate lengths, filtered according to the desired decay times $\tau(\omega)$, mixed via an orthonormal mixing matrix, and fed back.

However, when modeling a particular room impulse response, the psychoacoustically important impulse response onset is not preserved. To overcome this difficulty, Stewart and Murphy [35] proposed a hybrid structure. A short convolutional section exactly reproduces the reverberation onset, while an efficient FDN structure generates the late field with a computational cost that does not depend on the reverberation decay time. Stated mathematically, the hybrid reverberator impulse response is the sum of that of the convolutional section $c(t)$ and that of the FDN section $d(t)$

$$
h(t)=c(t)+d(t)
$$

The idea is then to adjust $c(t)$ and $d(t)$ so that the system impulse response $h(t)$ psychoacoustically approximates the measured impulse response $m(t)$. The convolutional section may be taken directly from the measured impulse response onset, and the equalization and decay rates of the FDN designed to match those of the measured late field response. In doing this, however, two issues arise: one having to do with the duration of the convolutional onset, and the other with the cross-fade between the convolutional and FDN sections. A quantity measuring closeness to Gaussian statistics called the normalized echo density (NED) has been shown to predict perceived echo density. In [36], the convolutional onset duration was given by the time at which the measured and FDN impulse responses achieve the same NED.

Regarding controlling the energy profiles of the onset and FDN components during the transition between the two, reference [36] suggests unrolling the loop of the FDN several times so that its impulse response energy onset is gradual. The convolutional response $c(t)$ is then windowed so that when it is summed with the FDN response $d(t)$, the resulting smoothed energy profile matches that of the measured impulse response. While this method is very effective, additional computational and memory resources are used in unrolling the loop. In [37], a constant-power cross-fade is achieved by simply subtracting the unwanted portion of the FDN response $d(t)$ from the convolutional response $c(t)$.

The EMT 140 plate reverberator is a widely used electromechanical reverberator, first introduced in the late 1950s. The EMT 140 consists of a large, thin, resonant plate mounted under tension. A driver near the plate center produces transverse mechanical disturbances which propagate over the plate surface, and are detected by pickups located toward the plate edges. A damping plate is positioned near the signal plate and is used to control the low-frequency decay time (see, e.g., [36]).

Bilbao [38] and Arcas and Chaigne [39] have explored the physics of plates and have developed finite difference schemes for simulating their motion. However, there are settings in which these schemes are impractical, and for real-time implementation as a (linear, time-invariant) reverberation effect, an efficient hybrid reverberator is useful. Here, the convolutional portion of the hybrid reverberator captures the distinctive whip-like onset of the plate impulse response, while the FDN reproduces the late-field decay, fixing reverberation time as a function of the damping plate setting.

3.3. Switched Convolution Reverberator. Both convolutional and delay line-based reverberators have significant memory requirements, convolutional reverberators needing a $60 \mathrm{~dB}$ decay time worth of samples and delay network reverberators requiring on the order of a second or two of sample memory. A comb filter structure requires little memory and may easily be designed to produce a pattern of echos having the desired equalization and decay rate. However, it has a constant, small echo density of one arrival per delay line length. This may be improved by adding a convolution with a noise sequence to the output. The resulting structure produces the desired echo density and impulse response spectrogram, and uses little memory-on the order of a few tenths of a second. The difficulty is that its output contains an undesired periodicity at the comb filter delay line length. As proposed in [40] and developed in [41], the periodicity may be reduced significantly by changing or "switching" the noise filter impulse response over time. Furthermore, by using velvet noise - a sequence of $\{+1,0,-1\}$ samples [40]an efficient time-domain implementation is possible.

A hybrid switched convolution reverberator was developed in [42] for efficiently matching the psychoacoustics of 
a measured impulse response. As above, the convolutional portion of the system is taken from the impulse response onset. However, here, the switched convolution reverberator noise sequences are drawn from the measured impulse response itself. In this way, the character of the measured late field is retained.

3.4. Spring Reverberators. Springs have been long used to delay and reverberate audio-bandwidth signals. Hammond introduced the spring reverberator in the late 1930s to enhance the sound of his electronic organs [43], and, since the 1960s with the introduction of torsionally driven tensioned springs [44], they have been a staple of guitar amps.

Modern spring reverbs consist of one or more springs held under tension, and they are driven and picked up torsionally from the spring ends. Spring mechanical disturbances propagate dispersively, and the primary torsional and longitudinal modes propagate low frequencies faster than high ones. Bilbao and Parker [45] have developed finite difference methods based on Wittrick's treatment of helical coils [46], generating accurate simulations. An efficient approximation, using the dispersive filter design method described in [47] is presented in [48]. There, a bidirectional waveguide implements the attenuation and dispersion seen by torsional waves travelling along the spring. A similar structure was used in [49] to model wave propagation along a Slinky. In addition, an FDN structure was proposed in which each delay line was made dispersive.

This model does not include the noise-like "wash" component of the impulse response, which may be the result of spring imperfections. In [50], an efficient waveguide-type model is described in which a varying delay generates the desired "wash." Additionally, a simple, noniterative design of high-order dispersive filters based on spectral delay filters was proposed in [50].

3.5. Delay Effects. The Leslie speaker, a rotating horn housed in a small cabinet [51-54], was often paired with a Hammond $\mathrm{B} 3$ organ. As the horn rotates, the positions of the direct path and reflections change, resulting in a varying timbre and spreading of the spectral components, due to Doppler shifts. Approaches to emulating the Leslie include separately modeling each arrival with an interpolated write according to the horn's varying position, and a biquad representing the horn radiation pattern [52]. In another recent approach [54], impulse responses are tabulated as a function of horn rotation angle. As the horn rotates, a timevarying FIR filter is applied to the input, with each filter tap drawn from a different table entry according to the horn's evolving rotational state. Rotation rates well into the audio bands were produced.

Tape delays, including the Maestro Echoplex and Roland SpaceEcho, are particularly challenging to model digitally. Their signal flow is simple and includes a delay and feedback. The feedback is often set to a value greater than one, causing the unit to oscillate, repeatedly amplifying the applied input or noise in the system. While the feedback loop electronics includes a saturating nonlinearity, much of the sonic character of these units arises from the tape transport mechanism, which produces both quasiperiodic and stochastic components, as described in $[55,56]$. Finally, it should be pointed out that the Echoplex uses a moveable record head to control the delay. The record head is easily moved faster than the tape speed, resulting in a "sonic boom". In [55], an interpolated write using a time-varying FIR antialiasing filter is proposed to prevent aliasing of this infinite-bandwidth event.

Bucket brigade delay lines have been widely used in chorus and delay processors since the 1970s. A sample and hold was used with a network of switched capacitors to delay an input signal according to an externally applied clock. However, as the charge representing the input signal is transferred from one capacitor to the next, a small amount bleeds to adjacent capacitors, and the output acquires a mild low-pass characteristic. In addition, while the charge is propagating through the delay line, it decays to the substrate. In this way, louder signals are distorted. A physical model of the device is presented in [57].

\section{Tube Amplifier Emulation}

Digital emulation of tube amplifiers has become an active area of commercial and academic interest during the last fifteen years [58]. The main goal in tube emulation is to produce flexible and realistic guitar amplifier simulation algorithms, which faithfully reproduce the sonic characteristics and parametric control of real vintage and modern guitar amplifiers and effects. Furthermore, these digital models should be computationally simple enough so that several instances could be run simultaneously in real-time. A recent review article [58] made an extensive survey of the existing digital tube amplifier emulation methods. The objective of the present section is to summarize the emulation approaches published after the aforementioned review.

4.1. Custom Nonlinear Solvers. Macak and Schimmel [59] simulate the diode limiter circuit, commonly found in many guitar distortion effects. They start with devising a first-order IIR filter according to the linearized equivalent circuit, after which the nonlinear effects are introduced by allowing the variation of the filter coefficients. The implicit nonlinear relation between the filter coefficients and its output is tackled using two alternative approaches. In the first approach, an additional unit delay is inserted into the system by evaluating the filter coefficients using the filter output at the previous sample. Obviously, this creates a significant error when the signal is changing rapidly, as can happen at high input levels, resulting in saturation. Thus, the first approach needs a high sampling rate to perform correctly, so that the signal value and system states do not change much between successive samples. The second approach is to solve the implicit nonlinearity using the Newton-Raphson method and use the previous filter output only as an initial estimate for the solver. Additionally, a nonlinear function is added for 


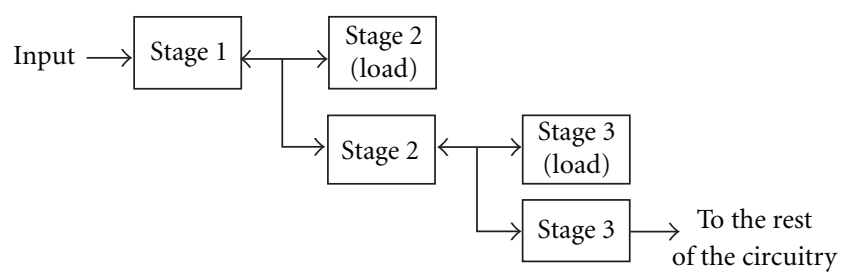

Figure 3: The preamplifier structure used in [60]. The interstage loading effects are approximated by simulating a pair of amplifier stages together and reading the output in between them. Thus, the latter stage of this pair acts simply as a load for the first stage and does not produce output.

saturating the estimate in order to accelerate the convergence of the iteration.

In a later article [60], Macak and Schimmel introduce an ordinary differential equation- (ODE-) based real-time model of an entire guitar amplifier. Although some parts of the amplifier are clearly oversimplified (ideal output transformer, constant power amplifier load, ideal impedance divider as the cathode follower), it is one of the most complete real-time amplifier models published in academic works. The ODEs for the tube stages are discretized using the backwards Euler method, and the implicit nonlinearities are approximated using the present input value and the previous state. The individual tube nonlinearities are modeled using Koren's equations [61], and the tone stack is implemented as reported in [62]. The algorithm is reportedly implemented as a VST-plugin.

The correct modeling of the mutual coupling between amplifier stages is important for realistic emulation, but efficient real-time simulation of this is a difficult task. On the one hand, a full circuit simulation of the amplifier circuitry provides a very accurate, although computationally inefficient model. On the other hand, a block-based cascade model with unidirectional signal flow can be implemented very efficiently, but is incapable of modeling the coupling effects.

An interesting hybrid approach has been used in [60], where the mutual coupling between the preamp triode stages is simulated by considering each pair of cascaded stages separately. For example, the output of stage 1 is obtained by simulating the cascaded stages 1 and 2 together, while the output is read in between the stages, as illustrated in Figure 3. Thus, the output of stage 2 is not used at this point, and the stage 2 is only acting as a load for stage 1 . The output of stage 2 is similarly obtained by simulating the cascade of stages 2 and 3 and reading the output between them. Interestingly, a similar modeling approach has been used in a recent commercial amplifier emulator [63].

4.2. State-Space Models. A promising state-space modeling technique for real-time nonlinear circuit simulation, the DK method, has been presented by Yeh and colleagues [64, 65]. It is based on the $\mathrm{K}$ method [66] introduced by Borin and others in 2000, and augments it by automating the model creation. Furthermore, the DK method discretizes the state elements prior to solving the system equations in order to avoid certain computability problems associated with the $\mathrm{K}$ method. The nonlinear equations are solved during runtime using Newton-Raphson iteration. In practice, with the DK method, the designer can obtain a digital model of a circuit simply by writing its netlist-a well-known textual representation of the circuit topology-and feeding it to the model generator.

Interestingly, the DK method allows the separation of the nonlinearity from the memory elements, removing the need for run-time iteration and thus allowing an efficient real-time implementation using look-up tables. However, for the memory separation to work properly, the circuit parameters should be held fixed during the simulation, thus disabling run-time control of the knobs on the virtual system. Alternatively, control parameter variations can be incorporated into the static nonlinearity by increasing its dimension, which makes the look-up table interpolation more challenging.

A variation to the DK method has been introduced by Dempwolf et al. [67]. In their paper, the system equations are derived manually, leading to more compact matrix representations. Also the discretization procedure is different. As a result, the method described in [67] is computationally less expensive than the DK method, but the model generation cannot be automated. The Marshall JCM900 preamp circuit is used as a case study in [67], and the simulation results show a good graphical and sonic match to measured data.

Another state-space representation for the 12AX7 triode stage is proposed by Cohen and Hélie [68], along with a comparison of the traditional static model and a novel dynamic model for the triode tube. In particular, Koren's static tube model [61] is augmented by adding the effect of stray capacitance between the plate and the grid, a source of the Miller effect in the amplifier circuit. An implicit numerical integration scheme is used for ensuring convergence, and the algorithm is solved using the Newton-Raphson method. The preamplifier model has been implemented as a real-time VST-plugin. A single-ended guitar power amplifier model using a linear output transformer has been reported in [69]. The pentode tube is simulated using Koren's equations [61], and the same state-space approach as in [68] is chosen for modeling. Also in [69], the simulation is implemented in real-time as a VST-plugin.

4.3. Wave-Digital Filter Models. The usability of wave digital filters (WDFs) in the virtual analog context is discussed by De Sanctis and Sarti in [70]. Importantly from the viewpoint of amp emulation, different strategies for coping with multiple nonlinearities and global feedback are reviewed. Traditionally, implementing a circuit with multiple nonlinear elements using WDFs requires special care. In [70], it is suggested that the part of the circuit containing multiple nonlinearities would be implemented as a single multiport nonlinearity, and the computability issues would be dealt inside this multiport block, for example using iterative techniques. This would essentially sacrifice some of the modularity of the WDF representation for easier computability. The consolidation of linear and time-invariant WDF elements 
as larger blocks for increasing computational efficiency is suggested already in an earlier work by Karjalainen [71].

A new WDF model of a triode stage has been introduced in [72]. In contrast to the previous WDF triode stage [73], this enhanced real-time model is capable of also simulating the triode grid current, thus enabling the emulation of phenomena such as interstage coupling and blocking distortion [74]. The plate-to-cathode connection is simulated using a nonlinear resistor implementing Koren's equations [61], while the grid-to-cathode connection is modeled with a tube diode model. The implicit nonlinearities are solved by the insertion of unit delays, so that there is no need for runtime iteration. Although the artificial delays theoretically compromise the modeling accuracy and model stability, in practice the simulation results show an excellent fit to SPICE simulations, and instability issues are not encountered for realistic signal levels.

The output chain of a tube amplifier, consisting of a single-ended-triode power amplifier stage, output transformer and a loudspeaker, is modeled using WDFs in [75]. The power amplifier stage uses the same triode model as in [72], thus allowing the simulation of the coupling between the power amp and loudspeaker. Linear equivalent circuits are devised for the transformer and loudspeaker, and the component values are obtained from datasheets and electrical measurements. The simulation is implemented as a computationally efficient fully parametric real-time model using the BlockCompiler software [76], developed by Matti Karjalainen.

4.4. Distortion Analysis. Since tube amplifier emulators are designed to mimic the sonic properties of real amplifiers and effects units, it is important for the system designer to be able to carefully measure and analyze the distortion behavior of real tube circuits. Although comparisons are typically done by subjective listening, objective methods for distortion analysis in tube amp emulation context have recently been reported [77-80]. In [77], the parameter variations on a highly simplified unidirectional model of a tube amp with static nonlinearities were studied using the exponential sweep analysis $[81,82]$. In particular, the shape of the static nonlinear curves and filter magnitude responses were individually varied, and the resulting effects on the output spectra with up to nine harmonic distortion components were analyzed.

In $[79,80,83]$, Novák and colleagues use the exponential sweep analysis in creating nonlinear polynomial models of audio devices. More specifically, the nonlinear model, called the generalized polynomial Hammerstein structure, consists of a set of parallel branches with a power function and a linear filter for each harmonic component. In [79], an audio limiter effect is simulated, while two overdrive effects pedals are analyzed and simulated in [80]. Reference [83] models an overdrive pedal using a parallel Chebyshev polynomial and filtering structure.

In [78], a software tool for distortion analysis is presented and a VOX AC30 guitar amplifier together with two commercial simulations are analyzed and compared.
The tool has five built-in analysis functions for measuring different aspects of nonlinear distortion, including the exponential sweep and dynamic intermodulation distortion analysis [84], and additional user-defined analysis techniques can be added using Matlab. The software is freely available at http://www.acoustics.hut.fi/publications/papers/DATK.

Finally, the use of nonlinear signal shaping algorithms has also been re-evaluated in view of modern analysis and modeling methods in [85]. Here the technique of phaseshaping is studied as an alternative to the more traditional nonlinear waveshaping algorithms. Employing a recent spectral analysis method, the Complex Spectral Phase Evolution (CSPE) algorithm, the distortion characteristics of overdrive effects are analyzed [86] and polynomial descriptions of phase and wave shaping functions are obtained from phase and amplitude harmonic data. The method outlined in that work is capable of reproducing distortion effects both in terms of their spectrum and waveform outputs.

\section{Digital Voltage-Controlled Filters}

The voltage-controlled filter (VCF) is a famous paradigm in real-time sound processing. Not only has it been recognized as a milestone in the history of electronic music, but in an attempt to reformulate the challenging solutions in its architecture in the digital domain, the various discrete-time models that were proposed in the last fifteen years to simulate the VCF have given rise to a curious thread of interesting realizations.

Developed originally by Moog [87], the VCF is composed of an RC ladder whose resistive part is formed by four transistor pairs in a differential configuration. These transistors are kept forward biased by a current source, which sets the cutoff frequency of the filter. The ladder's output is fed back to its input via a high-impedance amplifier in a way that generates, in the cutoff region, oscillations whose amplitude and persistence depend on a variable resistance that controls the amount of feedback. In the limit of maximum feedback, the VCF becomes an oscillator ringing at the cutoff frequency irrespective of the input.

Both the bias current and the variable resistance are user controls in Moog synthesizers, the former provided by DC signal generators and low-frequency oscillators, as well as by external signal sources, the latter by simply varying the resistance through a knob. Sometimes musicians have controlled the filter behavior by feeding musical signals of sufficient amplitude that the bias current is affected and the cutoff is varied in a complex interplay between synthesis and control. An analogous effect is produced when the injected currents contain frequency components that are high enough to reach the filter output.

Finally, the VCF response is affected by the input amplitude due to the many solid-state components in the circuitry. Large amplitude signals are in fact distorted by the transistors, giving rise to the characteristic nonlinear behavior of this filter. A similar, but not identical, behavior was exhibited by a VCF clone on board the EMS synthesizers, employing diodes instead of transistors in the RC ladder [89]. 
In conclusion, the VCF has compelling ingredients that make its simulation in the discrete-time especially interesting: (i) nonlinear behavior and (ii) two-dimensional continuous control, exerted by both parameter changes (i.e., the variable resistance governing the oscillatory behavior) and control signals (the bias current setting the cutoff point). As a result, it established a paradigm in virtual analog modeling.

5.1. Linear Digital VCFs. Even the reproduction of the VCFs linear behavior has to deal with the two-dimensional control space and its effects in the output. The problem can be further simplified by collapsing the current-based control mechanism into a scalar parameter of cutoff frequency. Such simplifications lead to the following transfer function:

$$
H(s)=\frac{\{G(s)\}^{4}}{1+k\{G(s)\}^{4}}=\frac{1}{k+\left\{1+s / \omega_{c}\right\}^{4}},
$$

in which frequency variable $\omega_{c}$ sets the cutoff frequency and feedback gain $k$ determines the oscillatory behavior (i.e., resonance). The function $G(s)=\omega_{c} /\left(\omega_{c}+s\right)$ models every single step of the ladder. Figure 4 shows, in dashed lines, typical magnitude responses of the analog Moog VCF obtained by plotting $|H(j \omega)|$ in audio frequency as by (4) and, in solid lines, spectra of discrete-time impulse responses after bilinear transformation of $H(s)$ into $H(z)$ at $44.1 \mathrm{kHz}$, respectively, for gains $k$ equal to $1,2,3$, and 4 . All responses have been plotted for cutoff frequencies $f_{c}=\omega_{c} /(2 \pi)$ equal to $0.1,1$, and $10 \mathrm{kHz}$ [88].

Stilson and Smith, in their pioneering approach to the linear modeling of the VCF [90], showed that the accurate real-time computation of (4) in discrete time is problematic. In fact, $k$ and $\omega_{c}$ merge into a bidimensional nonlinear map while moving to the digital domain. On the other hand, approximations of $H(s)$ aiming at maintaining such parameters decoupled in the discrete-time domain lead to inaccurate responses as well as to mismatches of the cut-off frequency and persistence of the oscillations compared to the analog case.

A step ahead in the linear VCF modeling has been achieved by Fontana, who directly computed the delayfree loop VCF structure arising from (4) and illustrated in Figure 5 for convenience [88]. By employing a specific procedure for the computation of delay-free loop filter networks [91], the couple $\left(\omega_{c}, k\right)$ in fact could be preserved in the discrete-time domain without mixing the two parameters together. In practice, this procedure allows to serialize the computation of the four identical transfer functions $G(z)$ obtained by the bilinear transformation of $G(s)$, independently of the feedback gain $k$. Three look-up tables and a few multiplications and additions are needed to obtain the feedback signal and the state variable values for each sampling interval. This way, an accurate response, an independent and continuous parametric control, and real-time operation are all achieved at a fairly low computational cost.

5.2. Nonlinear Digital VCFs. The introduction of nonlinearities complicates the problem to a large extent. When the nonlinear components, such as transistors or diodes, are modeled, the simulation can be developed starting from a plethora of VCF circuit approximations. The final choice often ends up on a mathematically tractable subset of such components, each modeled with its own degree of detail, allowing to establish a nonlinear differential state-space representation for the whole system.

Furthermore, different techniques exist to solve the nonlinear differential problem. Concerning the VCF, two fundamental paradigms have been followed: the functional paradigm, relying on Volterra expansions of the nonlinearities, and the circuit-driven paradigm, based on the algebraic solution of the nonlinear circuit. Both such paradigms yield solutions that must be integrated numerically. By solving simplified versions of the VCF in a different way, both of them are prone to various types of inaccuracies.

Huovilainen, who chose to use a circuit-driven approach [93], was probably the first to attempt a nonlinear solution of the VCF. He derived an accurate model of the transistorbased RC ladder as well as of the feedback circuit. On the other hand, while proposing a numerical solution to this model, he kept a fictitious unit delay in the resulting digital structure to avoid costs and complications of an implicit procedure for the feedback loop computation. The extra unit delay in the feedback loop creates an error in parameter accuracy, which increases with frequency. Huovilainen then uses low-order polynomial correction functions for both the cut-off frequency and the resonance parameter, thus still reaching a desired accuracy [92].

Figure 6 shows a simplified version of Huovilainen's nonlinear digital Moog filter, in which only one nonlinear function is used [92]. Huovilainen's full Moog ladder model contains five such functions: one for each first-order section and one for the feedback circuit. A hyperbolic tangent is used as the nonlinear function in [93]. In a real-time implementation, this would usually be implemented using a look-up table. Alternatively, another similar smoothly saturating function, such as a third-order polynomial, can be used instead. Huovilainen's nonlinear Moog filter selfoscillates, when the feedback gain is set to a value of one or larger. The saturating nonlinear function ensures that the system cannot become unstable, because signal values cannot grow freely. The simplified model of Figure 6 behaves essentially in the same manner as the full model, but small differences in their output signals can be observed. It remains as an interesting future study to test with which input signals and parameter setting their minor differences could be best heard.

The Volterra approach was proposed by Hélie in 2006 [95]. This approach requires a particular ability to manipulate Volterra kernels and to manage their instability in presence of heavy distortion. Indeed, high distortion levels can be generated by the VCF when fed large amplitude inputs and for high values of $k$, that is, when the filter is set to operate like a selective resonator or like an oscillator. In a more recent development proposed by the same author [96], sophisticated ad-hoc adaptations of the Volterra kernels were set in an aim to model the transistors' saturation on a sufficiently large amplitude range. 


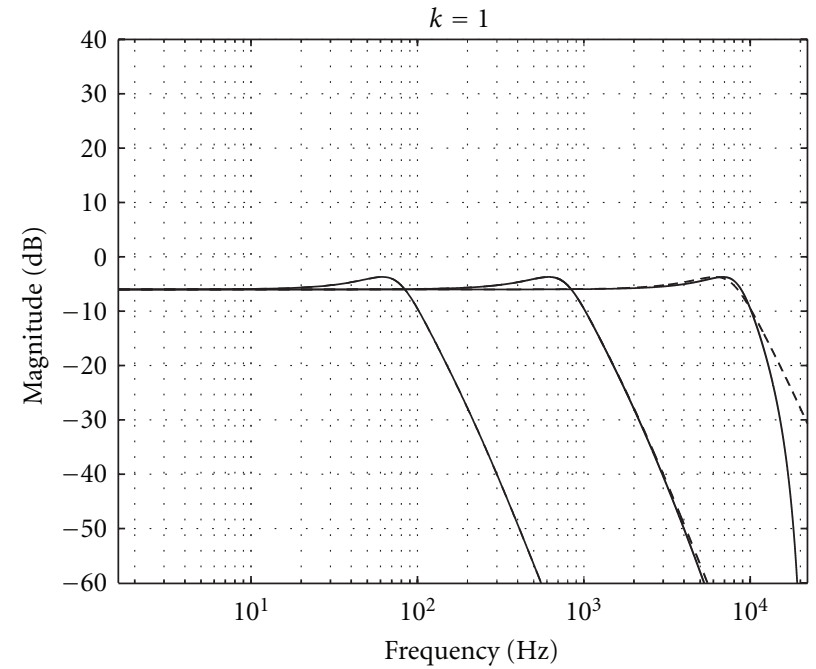

(a)

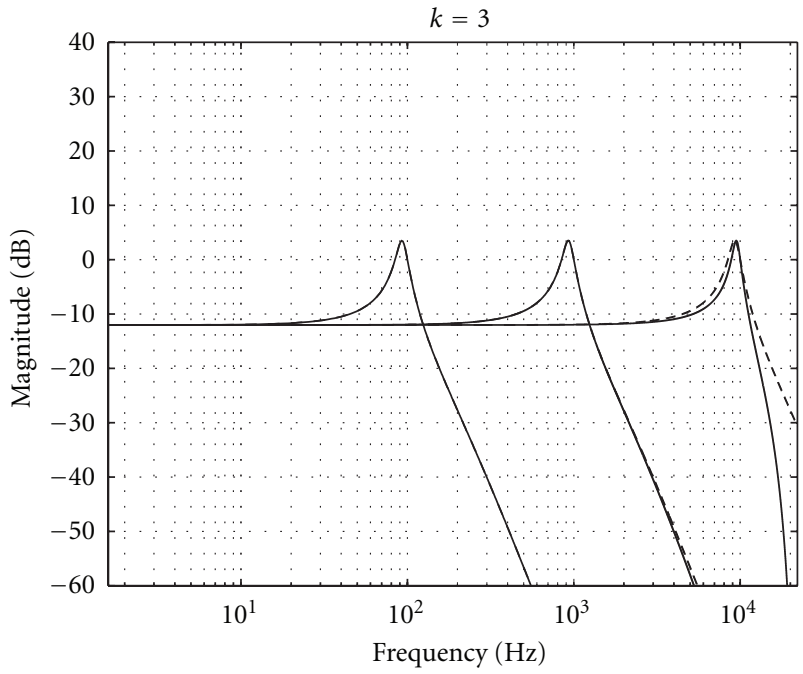

(c)

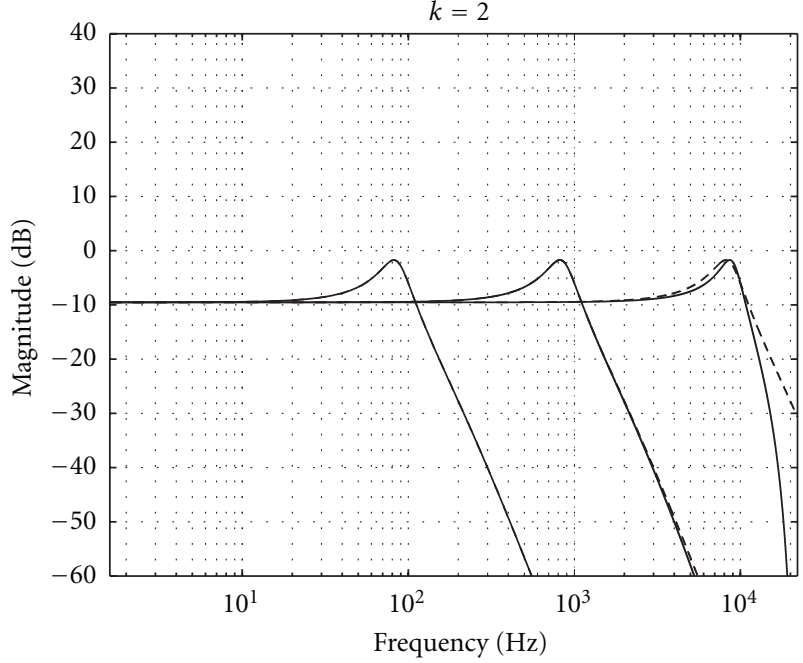

(b)

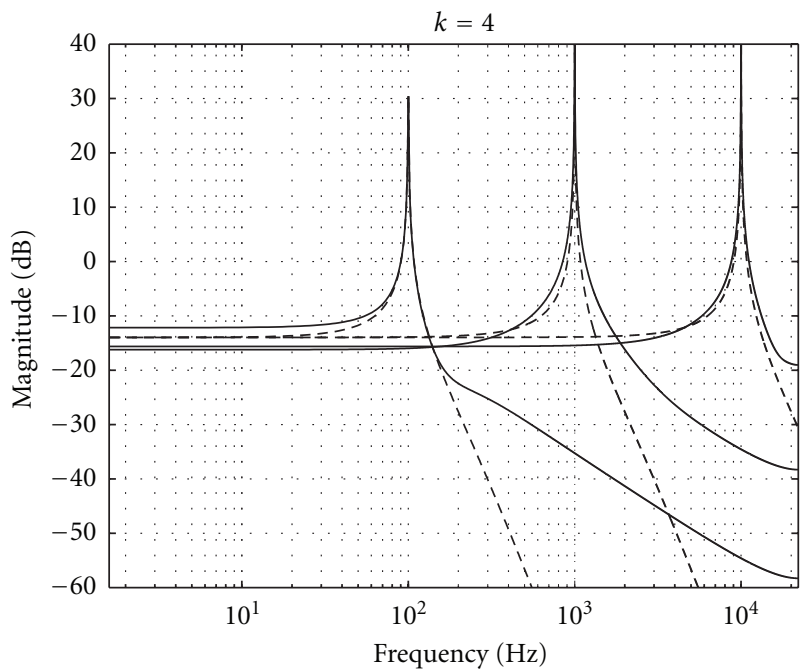

(d)

FIGURE 4: Magnitude responses of the analog linear Moog VCF (dashed line) and its digital version obtained by bilinear transformation at $44.1 \mathrm{kHz}$ (solid line). Cut-off frequencies equal to $0.1,1$, and $10 \mathrm{kHz}$ are plotted in each diagram [88].

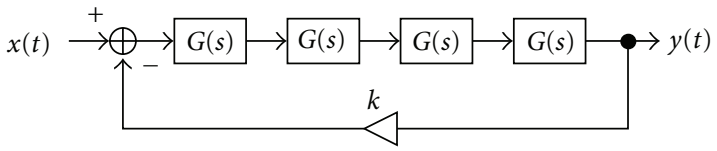

FIgURE 5: Delay-free loop filter structure of the VCF.

In 2008, Civolani and Fontana devised a nonlinear statespace representation of the diode-based EMS VCF out of an Ebers-Moll approximation of the driving transistors [97]. This representation could be computed in real time by means of a fourth-order Runge-Kutta numerical integration of the nonlinear system. The model was later reformulated in terms of a passive nonlinear analog filter network, which can readily be turned into a passive discrete-time filter network through

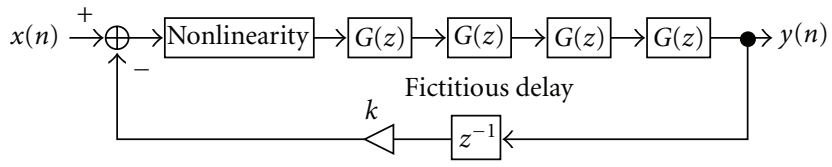

Figure 6: A simplified version of Huovilainen's nonlinear digital Moog filter [92].

any analog-to-digital transformation preserving passivity [94]. The delay-free loops in the resulting digital network were finally computed by repeatedly circulating the signal along the loop until convergence, in practice implementing a fixed-point numerical scheme.

Figure 7 provides examples of responses computed by the EMS VCF model when fed a large amplitude impulsive input [94]. On the left, the impulse responses for increasing 


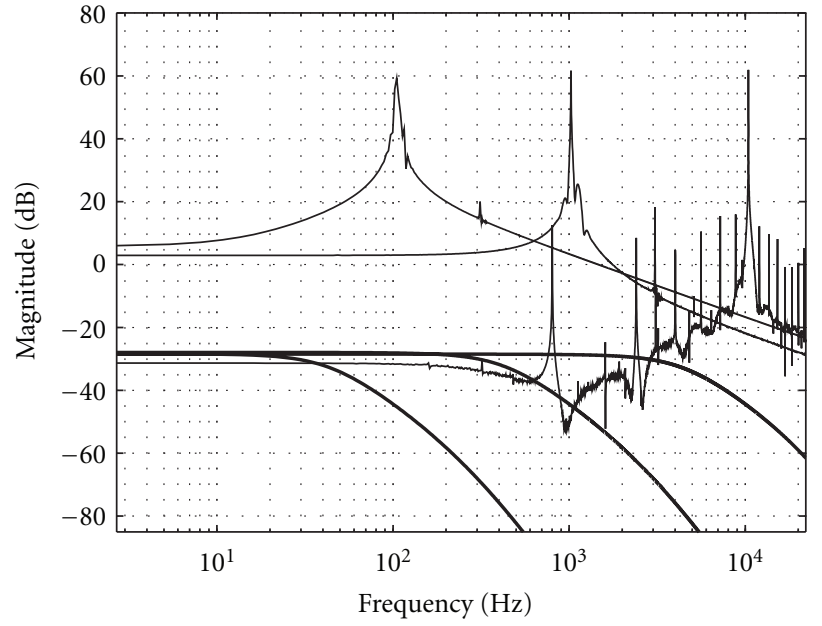

(a)

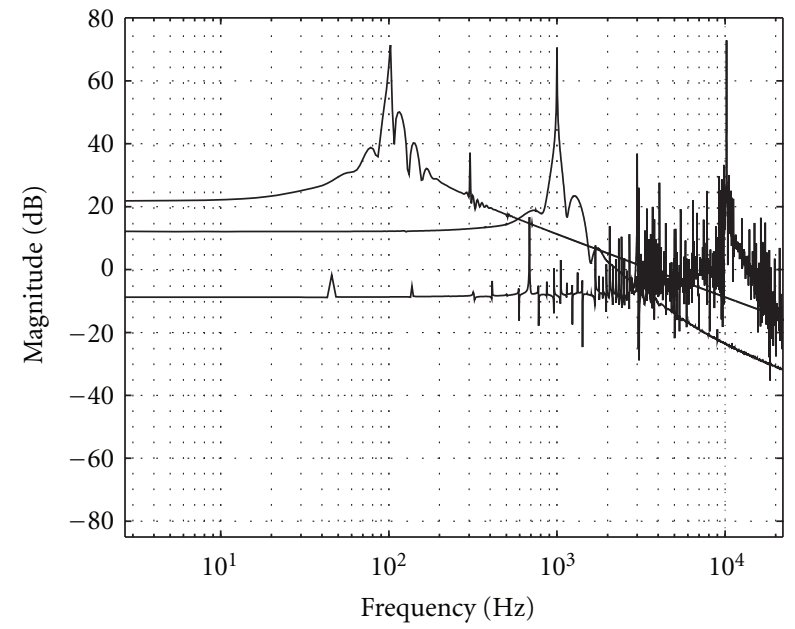

(b)

FIGURE 7: Magnitude responses of the EMS VCF model for a $1 \mathrm{~V}$ impulsive input and cutoff frequency set to $0.1,1$, and $10 \mathrm{kHz}$. (a) $k=0$ (bold), 8 (thin solid). (b) $k=11$. Sampling frequency set at $176.4 \mathrm{kHz}$ [94].

values of $k$ are illustrated at cut-off frequencies equal to $0.1,1$, and $10 \mathrm{kHz}$. On the right, the system behavior is illustrated with the same cut-off frequencies and a very high feedback gain. Comparison with Figure 4 helps to appreciate the contribution of the distortion components to the output, as well as their amount for changing values of the feedback gain parameter. Also for reasons that are briefly explained at the end of this section, Figure 7 does not include magnitude spectra of output signals measured on a real EMS VCF, due to the gap that still exists between the virtual analog model and the reference filter.

5.3. Current Issues. The current Java implementation for the PureData real-time environment [98] of the aforementioned delay-free loop filter network, obtained by bilinear transformation of the state-space representation of the EMS analog circuit [94], represents a highly sophisticated noncommercial realization of a VCF software architecture in terms of accuracy, moderate computational requirement, continuous controllability of both $\omega_{c}$ and $k$, and interoperability under all operating systems capable of running PureData and its $p d j$ libraries communicating with the Java Virtual Machine. In spite of all these desirable properties, the implementation leaves several issues open.

Especially some among such issues ask for a better understanding and consequent design of the digital filter.

(i) The bias current has been modeled so far in terms of a (concentrated) cut-off frequency parameter. As it has been previously explained, the analog VCF cutoff is instead biased by a current signal that flows across the filter together with the musical signal. The subtle, but audible nuances resulting from the continuous interplay between such two signals, can be reproduced only by substituting the cut-off frequency parameter in the state-space representation with one more system variable, accounting for the bias current.
Moreover, this generalization may provide a powerful control for musicians who appreciate the effects resulting from this interplay.

(ii) Although designed to have low or no interference with the RC ladder, the feedback circuit has a nonnegligible coupling effect with the feedforward chain. As we could directly assess on a diode-based VCF on board an EMS synthesizer during a systematic measurement session, the leaks affecting the feedback amplifier in fact give rise to responses that are often quite far from the "ideal" VCF behavior. Even when the feedback gain is set to zero, this circuit exhibits a nonnull current absorption that changes the otherwise stable fourth-order low-pass RC characteristics. Techniques aiming at improving the accuracy of the feedback amplifier would require to individually model at least some of its transistors, with consequences on the model complexity and computation time that cannot be predicted at the moment.

The next generation of virtual analog VCFs may provide answers to the above open issues.

\section{Synthesis and Processing Languages}

Languages for synthesis and processing of musical signals have been central to research and artistic activities in computer music since the late 1950s. The earliest digital sound synthesis system for general-purpose computers is MUSIC I by Max Mathews (1959), a very rudimentary program written for the IBM 704 computer [99], capable of generating a single triangular-shaped waveform. This was followed in quick succession by MUSIC II and III, introducing some of the typical programming structures found in all of today's systems, such as the table look-up oscillator [100]. These developments culminated in MUSIC IV (1963), which 
provided many of the elements that are found in modern systems. One of the most important ideas introduced by this program was the concept of modular synthesis components, which can be used to construct instruments for computer music performance defined in a score code. In particular, the principle of the unit generator (UG), on which all modern systems are based, was introduced in this system. UGs are the building blocks of synthesis environments, implementing the basic operations that are responsible for digital audio generation.

Another major step in the development of languages for synthesis and processing was the adoption of portable highlevel programming languages for their implementation. An early example of this was seen in MUSIC IVBF, a version of MUSIC IV written in FORTRAN at Princeton University in 1964. But it is Mathews' MUSIC V [101], also based on FORTRAN, that occupies a central place in the development of computer music for its widespread adoption, providing a model on which modern systems would be based.

Modern descendants from these systems include Csound 5 [102], Pure Data (PD) [103], and SuperCollider 3 (SC3) [104] as the most established and commonly used opensource systems. Other currently supported languages include Nyquist [105], also used for plugin scripting in the Audacity sound editor, and PWGL [106] which supports a comprehensive computer-aided composition system. Most of these environments are designed for real-time applications, although Csound was originally written as an offline processing program and indeed can still be used that way. Processing is generally done in a block-by-block basis, and UGs are provided in an object-oriented fashion. Csound and SC3 provide two basic rates of processing for control and audio signals, and PD provides a single rate (for audio) with control signals processed asynchronously by a messagepassing mechanism.

SC3 is actually based on two components, a programming language/interpreter, SCLang, and a sound synthesis engine, SCSynth. They are combined in a client-server structure, with the former issuing Open Sound Control (OSC) commands over an IP connection to the server. This also allows the synthesis engine to be used independently of the language interpreter, by any software capable of providing OSC output. SCLang is an object-oriented language that provides a symbolic representation of the UG entities residing in the server and allowing the user to create connections between these. The synthesis engine will, on receiving certain OSC messages, construct UG graphs and schedule processing accordingly. New UGs can be added to the system as dynamic modules, which are loaded by the server on startup. For these to be legal SCLang constructs, they also have to be provided an interface for the language. SC3 has been used by various research and artistic projects, such as the ones described in [107].

Unlike SC3, PD is a flowchart programming language. It provides a graphical interface that is used to create programs (also known as patches), although these can also be created as a plain text script (or indeed programmatically). Central to its operation is an object-oriented messagepassing mechanism. UGs are built to respond to particular types of messages with given methods. Messages are passed through wire connections between objects. For audio, a special type of UG is required, which will allow for audio input and/or output connections and also provide a method for a DSP message. This enables the object to register its processing routine with the systems audio processing scheduler, so that it is included in the DSP graph. As with SC3, UGs can be added to PD as dynamic modules that are either loaded at startup or, in certain cases, on demand. Given this relatively simple means of language extension, PD has also been adopted as system for the implementation and demonstration of new algorithms, as for instance in [108]. Finally, we should note that PD has a closed-source, commercially available, equivalent alternative, MAX/MSP [109].

Of these three systems, Csound is the longest in existence, having been developed in 1985 and released publicly in 1996. It has, however, been fundamentally modified for version 5, first released in 2006, which has brought its code base up-to-date with more recent programming practices. Effectively, Csound is a programming library, which can be used from various languages, for example, $\mathrm{C} / \mathrm{C}++$, Java, Python, Lua, Tcl, and so forth. As a synthesis system, it provides a text language for the creation of instruments from UGs and a compiler to generate DSP graphs from these. It has also a separate basic score language, which can be substituted or combined with other means of instrument instantiation. A number of frontends to the system exist, allowing it to be used in different contexts and applications. For signal processing research, it allows prototyping of new algorithms (e.g., filters and synthesis equations) in a simple and direct way, as well as sample-by-sample processing. Its integration with the Python language is particularly useful as it allows for the graphing and numerical libraries to be combined in scripts. In addition, for frequencydomain applications, it provides a special signal type that is used for stream processing. This is a very useful feature for the testing and implementation of real-time spectral applications. UGs can be added to the system via dynamic loadable libraries, allowing for the implementation of newlydeveloped algorithms, such as [8].

In addition to the MUSIC $\mathrm{N}$-derived systems described above, there is one further language of note. This is FAUST, a purely functional language for prototyping and implementation of audio processing algorithms [110]. It aims to be an efficient alternative to implementation languages such as $\mathrm{C}$ or C++. FAUST is better described as a signal processing, rather than a music, programming language. It is based on the abstraction of a signal processor as a mathematical function taking inputs and producing outputs. While not designed in the same vein, and with the same principles, as the ones discussed above, it nevertheless shares their modular approach, with structural elements that are analogous to UGs. FAUST shares the flowgraph approach that directly underpins flowchart languages such as PD (and indirectly, all other MUSIC N-derived languages), but provides an explicit formal semantic. Also, unlike other systems, it produces $\mathrm{C}++$ code (as opposed to running DSP graphs) for various targets: UGs for SC3, PD (/MaxMSP), Csound, and so 
forth; standalone programs with various audio I/O backends; and plugins of various formats. FAUST was designed with the aims of allowing rapid translation of algorithms and flowcharts into functional code and generation of efficient $\mathrm{C}++$ code, which is a very useful feature for real-time musical signal processing applications.

Finally, with the increased availability of multiple processor systems in general-purpose computers, systems have been developed to take advantage of these platforms. Two opposing approaches have been taken, representing different ideas of how parallelization should be achieved. These are represented typically by, on one side, a new version of SC3 (SuperNova) [111] and, on the other, by an experimental version of Csound, ParCS [112] and the OpenMP-based code output of FAUST [110]. The first approach follows the existing implementation of concurency in some programming languages, such as Occam [113], where the system provides constructs for users to parallelize portions of their code. The other approach is to let the parser decide automatically how the parallelization should be done, with the user having little or no role in determining it. This theory allows complete backwards compatibility with existing code and a seamless transition to newer hardware.

\section{Conclusion}

A selection of recent advances in musical effects processing and synthesis have been discussed in this paper. In particular, the advances in adaptive effects processing algorithms, synthesis, and processing languages, and digital emulation of tube amplifiers, voltage-controlled filters, and vintage delay and reverberation effects have been reviewed.

\section{Acknowledgment}

This work has been funded by the Aalto University and the Academy of Finland (Project no. 122815).

\section{References}

[1] U. Zölzer, Ed, DAFX_Digital Audio Effects, John Wiley \& Sons, New York, NY, USA, 2002.

[2] J. O. Smith, "Physical Audio Signal Processing," 2010, https:// ccrma.stanford.edu/ jos/pasp/.

[3] V. Välimäki, F. Fontana, J. O. Smith, and U. Zölzer, "Introduction to the special issue on virtual analog audio effects and musical instruments," IEEE Transactions on Audio, Speech and Language Processing, vol. 18, no. 4, pp. 713-714, 2010.

[4] V. Välimäki, J. Pakarinen, C. Erkut, and M. Karjalainen, "Discrete-time modelling of musical instruments," Reports on Progress in Physics, vol. 69, no. 1, pp. 1-78, 2006.

[5] V. Välimäki and A. Huovilainen, "Antialiasing oscillators in subtractive synthesis," IEEE Signal Processing Magazine, vol. 24, no. 2, pp. 116-125, 2007.

[6] J. Pakarinen, H. Penttinen, V. Välimäki et al., "Review of sound synthesis and effects processing for interactive mobile applications," Report 8, Department of Signal Processing and Acoustics, Helsinki University of Technology, 2009,
http://www.acoustics.hut.fi/publications/papers/MobileSynthAndFXReport/MobileSynthAndFXReport.pdf.

[7] C. Poepel and R. B. Dannenberg, "Audio signal driven sound synthesis," in Proceedings of the International Computer Music Conference, pp. 391-394, Barcelona, Spain, September 2005.

[8] V. Lazzarini, J. Timoney, and T. Lysaght, "The generation of natural-synthetic spectra by means of adaptive frequency modulation," Computer Music Journal, vol. 32, no. 2, pp. 922, 2008.

[9] J. Pekonen, "Coefficient modulated first-order allpass filter as distortion effect," in Proceedings of the International Conference on Digital Audio Effects, pp. 83-87, Espoo, Finland, September 2008.

[10] J. Kleimola, J. Pekonen, H. Penttinen, V. Välimäki, and J. S. Abel, "Sound synthesis using an allpass filter chain with audio-rate coefficient modulation," in Proceedings of the International Conference on Digital Audio Effects, Como, Italy, September 2009.

[11] C. M. Cooper and J. S. Abel, "Digital simulation of brassiness and amplitude-dependent propagation speed in wind instruments," in Proceedings of the International Conference on Digital Audio Effects, Graz, Austria, September 2010.

[12] V. Verfaille, U. Zölzer, and D. Arfib, "Adaptive digital audio effects (A-DAFx): a new class of sound transformations," IEEE Transactions on Audio, Speech and Language Processing, vol. 14, no. 5, pp. 1817-1831, 2006.

[13] T. Stilson, "General weirdness with the Karplus-Strong string," in Proceedings of the International Computer Music Conference, Banff, Canada, 1995.

[14] T. I. Laakso, V. Välimäki, M. Karjalainen, and U. K. Laine, "Splitting the unit delay: tools for fractional delay filter design," IEEE Signal Processing Magazine, vol. 13, no. 1, pp. 30-60, 1996.

[15] V. Lazzarini, J. Timoney, and T. Lysaght, "Asymmetricspectra methods for adaptive FM synthesis," in Proceedings of the International Conference on Digital Audio Effects (DAFx '08), pp. 233-240, Espoo, Finland, September 2008.

[16] V. Lazzarini, J. Timoney, and T. Lysaght, "Nonlinear distortion synthesis using the split-sideband method, with applications to adaptive signal processing," Journal of the Audio Engineering Society, vol. 56, no. 9, pp. 684-695, 2008.

[17] V. Lazzarini and J. Timoney, "Theory and practice of modified frequency modulation synthesis," Journal of the Audio Engineering Society, vol. 58, no. 6, pp. 459-471, 2010.

[18] V. Välimäki, J. S. Abel, and J. O. Smith, "Spectral delay filters," Journal of the Audio Engineering Society, vol. 57, no. 7-8, pp. 521-531, 2009.

[19] V. Lazzarini, J. Timoney, J. Pekonen, and V. Välimäki, "Adaptive phase distortion synthesis," in Proceedings of the International Conference on Digital Audio Effects, Como, Italy, September 2009.

[20] V. Välimäki, T. Tolonen, and M. Karjalainen, "Signaldependent nonlinearities for physical models using timevarying fractional delay filters," in Proceedings of the International Computer Music Conference, pp. 264-267, Ann Arbor, Mich, USA, October 1998.

[21] R. Msallam, S. Dequidt, S. Tassart, and R. Caussé, "Physical model of the trombone including nonlinear propagation effects," in Proceedings of the International Symposium on Musical Acoustics, vol. 2, pp. 419-424, Edinburgh, UK, 1997.

[22] S. Tassart, R. Msallam, P. Depalle, and S. Dequidt, "A fractional delay application: time-varying propagation speed 
in waveguides," in Proceedings of the International Computer Music Conference, pp. 256-259, Thessaloniki, Greece, September 1997.

[23] R. Msallam, S. Dequidt, R. Caussé, and S. Tassart, "Physical model of the trombone including nonlinear effects. Application to the sound synthesis of loud tones," Acta Acustica united with Acustica, vol. 86, no. 4, pp. 725-736, 2000.

[24] T. Tolonen, V. Välimäki, and M. Karjalainen, "Modeling of tension modulation nonlinearity in plucked strings," IEEE Transactions on Speech and Audio Processing, vol. 8, no. 3, pp. 300-310, 2000.

[25] J. Pakarinen, V. Välimäki, and M. Karjalainen, "Physicsbased methods for modeling nonlinear vibrating strings," Acta Acustica United with Acustica, vol. 91, no. 2, pp. 312 325, 2005.

[26] J. R. Pierce and S. A. van Duyne, "A passive nonlinear digital filter design which facilitates physics-based sound synthesis of highly nonlinear musical instruments," Journal of the Acoustical Society of America, vol. 101, no. 2, pp. 1120-1126, 1997.

[27] “Bill Putnam," 2011, http://en.wikipedia.org/wiki/Bill_Putnam.

[28] A. V. Oppenheim and R. W. Schafer, Discrete-Time Signal Processing, Pearson Prentice Hall, Upper Saddle River, NJ, USA, 3rd edition, 2010.

[29] W. G. Gardner, "Efficient convolution without input-output delay," Journal of the Audio Engineering Society, vol. 43, no. 3, pp. 127-136, 1995.

[30] D. S. McGrath, "Method and apparatus for filtering an electronic environment with improved accuracy and efficiency and short flow-through delay," US patent no. 5502747, Lake Technology Ltd., March 1996.

[31] G. Garcia, "Optimal filter partition for efficient convolution with short input/output delay," in Proceedings of the 113th AES Convention, October 2002, paper no. 5660.

[32] J. Hurchalla, "Low latency convolution in one dimension via two dimensional convolutions: an intuitive approach," in Proceedings of the 125th AES Convention, October 2008, paper no. 7634.

[33] M. R. Schroeder, "Natural sounding reverberation," Journal of the Audio Engineering Society, vol. 10, pp. 219-223, 1962.

[34] J.-M. Jot and A. Chaigne, "Digital delay networks for designing artificial reverberators," in Proceedings of the 90th AES Convention, Paris, France, 1991, preprint 3030.

[35] R. Stewart and D. Murphy, "A hybrid artificial reverberation algorithm," in Proceedings of the 122th AES Convention, May 2007, paper no. 7021.

[36] J. S. Abel, D. P. Berners, and A. Greenblatt, "An emulation of the EMT 140 plate reverberator using a hybrid reverberator structure," in Proceedings of the 127th AES Convention, New York, NY, USA, October 2009, paper no. 7928.

[37] A. Greenblatt, J. Abel, and D. Berners, "A hybrid reverberation crossfading technique," in Proceedings of the International Conference on Acoustics, Speech, and Signal Processing (ICASSP '10), pp. 429-432, Dallas, Tex, USA, 2010.

[38] S. Bilbao, "A digital plate reverberation algorithm," Journal of the Audio Engineering Society, vol. 55, no. 3, pp. 135-144, 2007.

[39] K. Arcas and A. Chaigne, "On the quality of plate reverberation,” Applied Acoustics, vol. 71, no. 2, pp. 147-156, 2010.

[40] M. Karjalainen and H. Järveläinen, "Reverberation modeling using velvet noise," in Proceedings of the 30th International Conference on Intelligent Audio Environments (AES '07), Saariselkä, Finland, March 2007.
[41] K.-S. Lee, J. S. Abel, V. Välimäki, and D. P. Berners, "The switched convolution reverberator," in Proceedings of the 127th AES Convention, New York, NY, USA, October 2009, preprint 7927.

[42] K.-S. Lee, N. J. Bryan, and J. S. Abel, "Approximating measured reverberation using a hybrid fixed/switched convolution structure," in Proceedings of the 13th International Conference on Digital Audio Effects (DAFx'10), Graz, Austria, September 2010.

[43] L. Hammond, "Electrical musical instrument," US patent no. 2230836, February 1941.

[44] A. C. Young, "Artificial reverberation unit," US patent no. 3106610, October 1963.

[45] S. Bilbao and J. Parker, "A virtual model of spring reverberation," IEEE Transactions on Audio, Speech and Language Processing, vol. 18, no. 4, pp. 799-808, 2010.

[46] W. H. Wittrick, "On elastic wave propagation in helical springs," International Journal of Mechanical Sciences, vol. 8, no. 1 , pp. 25-47, 1966.

[47] J. S. Abel and J. O. Smith, "Robust design of very high-order dispersive allpass filters," in Proceedings of the International Conference on Digital Audio Effects (DAFx '06), pp. 13-18, Montreal, Canada, September 2006.

[48] J. S. Abel, D. P. Berners, S. Costello, and J. O. Smith, "Spring reverb emulation using dispersive allpass filters in a waveguide structure," Journal of the Audio Engineering Society, vol. 54, p. 1277, 2006, presented at the 121th Convention of the Audio Engineering Society.

[49] J. Parker, H. Penttinen, S. Bilbao, and J. S. Abel, "Modeling methods for the highly dispersive slinky spring: a novel musical toy," in Proceedings of the 13th International Conference on Digital Audio Effects (DAFx'10), Graz, Austria, September 2010.

[50] V. Välimäki, J. Parker, and J. S. Abel, "Parametric spring reverberation effect," Journal of the Audio Engineering Society, vol. 58, no. 7-8, pp. 547-562, 2010.

[51] D. Leslie, "Rotatable tremulant sound," US patent 2489653, 1949.

[52] J. Smith, S. Serafin, J. Abel, and D. Berners, "Doppler simulation and the Leslie," in Proceedings of the 5th International Conference on Digital Audio Effects (DAFx '02), Hamburg, Germany, September 2002.

[53] R. Kronland-Martinet and T. Voinier, "Real-time perceptual simulation of moving sources: application to the Leslie Cabinet and 3D sound immersion," EURASIP Journal on Audio, Speech, and Music Processing, vol. 2008, Article ID 849696, 10 pages, 2008.

[54] J. Herrera, C. Hanson, and J. S. Abel, "Discrete time emulation of the Leslie speaker," in Proceedings of the 127th AES Convention, New York, NY, USA, October 2009, paper no. 7925.

[55] S. Arnardottir, J. S. Abel, and J. O. Smith, "A digital model of the Echoplex tape delay," in Proceedings of the 125th AES Convention, San Francisco, Calif, USA, October 2008.

[56] V. Välimäki, S. Bilbao, J. O. Smith, J. S. Abel, J. Pakarinen, and D. Berners, "Virtual analog effects," in DAFX: Digital Audio Effects, U. Zölzer, Ed., chapter 12, John Wiley \& Sons, New York, NY, USA, 2nd edition, 2011.

[57] C. Raffel and J. O. Smith, "Practical modeling of bucketbrigade device circuits," in Proceedings of the 13th International Conference on Digital Audio Effects (DAFx '10), Graz, Austria, September 2010. 
[58] J. Pakarinen and D. T. Yeh, "A review of digital techniques for modeling vacuum-tube guitar amplifiers," Computer Music Journal, vol. 33, no. 2, pp. 85-100, 2009.

[59] J. Macak and J. Schimmel, "Nonlinear circuit simulation using time-variant filter," in Proceedings of the International Conference on Digital Audio Effects, Como, Italy, September 2009.

[60] J. Macak and J. Schimmel, "Real-time guitar tube amplifier simulation using an approximation of differential equations," in Proceedings of the 13th International Conference on Digital Audio Effects (DAFx'10), Graz, Austria, September 2010.

[61] N. Koren, "Improved vacuum-tube models for SPICE simulations," Glass Audio, vol. 8, no. 5, pp. 18-27, 1996.

[62] D. T. Yeh and J. O. Smith, "Discretization of the '59 Fender Bassman tone stack," in Proceedings of the International Conference on Digital Audio Effects, pp. 1-6, Montreal, Canada, September 2006.

[63] T. Serafini, personal correspondence, 2009.

[64] D. T. Yeh, Digital implementation of musical distortion circuits by analysis and simulation, Ph.D. thesis, Stanford University, Palo Alto, Calif, USA, 2001, https://ccrma.stanford.edu/ dtyeh/papers/DavidYehThesissinglesided.pdf.

[65] D. T. Yeh, J. S. Abel, and J. O. Smith III, "Automated physical modeling of nonlinear audio circuits for real-time audio effects-part I: theoretical development," IEEE Transactions on Audio, Speech and Language Processing, vol. 18, no. 4, pp. 728-737, 2010.

[66] G. Borin, G. De Poli, and D. Rocchesso, "Elimination of delay-free loops in discrete-time models of nonlinear acoustic systems," IEEE Transactions on Speech and Audio Processing, vol. 8, no. 5, pp. 597-604, 2000.

[67] K. Dempwolf, M. Holters, and U. Zölzer, "Discretization of parametric analog circuits for real-time simulations," in Proceedings of the 13th International Conference on Digital Audio Effects (DAFx'10), Graz, Austria, September 2010.

[68] I. Cohen and T. Hélie, "Simulation of a guitar amplifier stage for several triode models: examination of some relevant phenomena and choice of adapted numerical schemes," in Proceedings of the 127th AES Convention, New York, NY, USA, 2009.

[69] I. Cohen and T. Hélie, "Real-time simulation of a guitar power amplifier," in Proceedings of the 13th International Conference on Digital Audio Effects (DAFx'10), Graz, Austria, September 2010.

[70] G. De Sanctis and A. Sarti, "Virtual analog modeling in the wave-digital domain," IEEE Transactions on Audio, Speech and Language Processing, vol. 18, no. 4, pp. 715-727, 2010.

[71] M. Karjalainen, "Efficient realization of wave digital components for physical modeling and sound synthesis," IEEE Transactions on Audio, Speech and Language Processing, vol. 16, no. 5, pp. 947-956, 2008.

[72] J. Pakarinen and M. Karjalainen, "Enhanced wave digital triode model for real-time tube amplifier emulation," IEEE Transactions on Audio, Speech and Language Processing, vol. 18, no. 4, pp. 738-746, 2010.

[73] M. Karjalainen and J. Pakarinen, "Wave digital simulation of a vacuum-tube amplifier," in Proceedings of the IEEE International Conference on Acoustics, Speech and Signal Processing (ICASSP '06), vol. 5, pp. 153-156, Toulouse, France, May 2006.

[74] R. Aiken, "What is blocking distortion?" internet article, 2006, http://www.aikenamps.com/BlockingDistortion.html.
[75] J. Pakarinen, M. Tikander, and M. Karjalainen, "Wave digital modeling of the output chain of a vacuum-tube amplifier," in Proceedings of the 12th International Conference on Digital Audio Effects (DAFx '09), Como, Italy, September 2009.

[76] M. Karjalainen, "BlockCompiler: efficient simulation of acoustic and audio systems," in Proceedings of the 114th AES Convention, Amsterdam, The Netherlands, March 2003.

[77] K. Dempwolf, M. Holters, S. Möller, U. Zölzer, and G. Hamburg, "The influence of small variations in a simplified guitar amplifier model," in Proceedings of the 12th International Conference on Digital Audio Effects (DAFx '09), Como, Italy, September 2009.

[78] J. Pakarinen, "Distortion analysis toolkit—a software tool for easy analysis of nonlinear audio systems," EURASIP Journal on Advances in Signal Processing, vol. 2010, Article ID 617325, 13 pages, 2010.

[79] A. Novák, L. Simon, F. Kadlec, and P. Lotton, "Nonlinear system identification using exponential swept-sine signal," IEEE Transactions on Instrumentation and Measurement, vol. 59, no. 8, pp. 2220-2229, 2009.

[80] A. Novák, L. Simon, and P. Lotton, "Analysis, synthesis, and classification of nonlinear systems using synchronized sweptsine method for audio effects," EURASIP Journal on Advances in Signal Processing, vol. 2010, Article ID 793816, 8 pages, 2010.

[81] A. Farina, "Simultaneous measurement of impulse response and distortion with a swept-sine technique," in Proceedings of the 108th AES Convention, Paris, France, February 2000, preprint 5093.

[82] J. Abel and D. P. Berners, "A technique for nonlinear system measurement," in Proceedings of the 121st AES Convention, San Francisco, Calif, USA, October 2006.

[83] A. Novák, L. Simon, P. Lotton, and J. Gilbert, "Chebyshev model and synchronized swept sine method in nonlinear audio effect modeling," in Proceedings of the 13th International Conference on Digital Audio Effects (DAFx '10), Graz, Austria, September 2010.

[84] E. Leinonen, M. Otala, and J. Curl, "A method for measuring transient intermodulation distortion (TIM)," Journal of the Audio Engineering Society, vol. 25, no. 4, pp. 170-177, 1977.

[85] J. Timoney, V. Lazzarini, A. Gibney, and J. Pekonen, “Digital emulation of distortion effects by wave and phase shaping methods," in Proceedings of the 13th International Conference on Digital Audio Effects (DAFx '10), pp. 419-422, Graz, Austria, September 2010.

[86] R. Garcia and K. Short, "Signal analysis using the complex spectral phase evolution (CSPE) algorithm," in Proceedings of the 120th AES Convention, Paris, France, May 2006.

[87] R. Moog, "A voltage-controlled low-pass high-pass filter for audio signal processing," in Proceedings of the 17th AES Convention, New York, NY, USA, October 1965, preprint 413.

[88] F. Fontana, "Preserving the structure of the Moog VCF in the digital domain," in Proceedings of the International Computer Music Conference, pp. 291-294, Copenhagen, Denmark, August 2007.

[89] T. E. Stinchcombe, "Analysis of the Moog transistor ladder and derivative filters," Tech. Rep., Freeserve, 2008, http://www.timstinchcombe.co.uk/.

[90] T. Stilson and J. O. Smith, "Analyzing the Moog VCF with considerations for digital implementation," in Proceedings of the International Computer Music Conference, Hong Kong, August 1996. 
[91] F. Fontana and F. Avanzini, "Computation of delay-free nonlinear digital filter networks: application to chaotic circuits and intracellular signal transduction," IEEE Transactions on Signal Processing, vol. 56, no. 10, pp. 4703-4715, 2008.

[92] V. Välimäki and A. Huovilainen, "Oscillator and filter algorithms for virtual analog synthesis," Computer Music Journal, vol. 30, no. 2, pp. 19-31, 2006.

[93] A. Huovilainen, "Nonlinear digital implementation of the Moog ladder filter," in Proceedings of the International Conference on Digital Audio Effects, Naples, Italy, October 2004.

[94] F. Fontana and M. Civolani, "Modeling of the EMS VCS3 voltage-controlled filter as a nonlinear filter network," IEEE Transactions on Audio, Speech and Language Processing, vol. 18, no. 4, pp. 760-772, 2010.

[95] T. Hélie, "On the use of Volterra series for real-time simulations of weakly nonlinear analog audio device: application to the Moog ladder filter," in Proceedings of the International Conference on Digital Audio Effects, pp. 7-12, Montreal, Canada, September 2006.

[96] T. Hélie, "Volterra series and state transformation for realtime simulations of audio circuits including saturations: application to the Moog ladder filter," IEEE Transactions on Audio, Speech and Language Processing, vol. 18, no. 4, pp. 747759, 2010.

[97] M. Civolani and F. Fontana, "A nonlinear digital model of the EMS VCS3 voltage-controlled filter," in Proceedings of the International Conference on Digital Audio Effects (DAFX '08), pp. 35-42, Espoo, Finland, September 2008

[98] M. Puckette, "Pure data," in Proceedings of the International Computer Music Conference, pp. 224-227, Thessaloniki, Greece, 1997.

[99] P. Manning, Electronic and Computer Music, Oxford University Press, Oxford, UK, 1989.

[100] M. V. Mathews, "The digital computer as a musical instrument," Science, vol. 142, no. 3592, pp. 553-557, 1963.

[101] M. V. Mathews, J. E. Miller, F. R. Moore, J. R. Pierce, and J. C. Risset, The Technology of Computer Music, MIT Press, Cambridge, Mass, USA, 1969.

[102] J. ffitch, "On the design of Csound 5," in Proceedings of the 3rd Linux Audio Conference, pp. 37-42, 2005.

[103] M. Puckette, The Theory and Technique of Electronic Music, World Scientific Press, 2007.

[104] J. McCartney, "Rethinking the computer music language: SuperCollider," Computer Music Journal, vol. 26, no. 4, pp. 61-68, 2002.

[105] R. B. Dannenberg, "Machine tongues XIX: Nyquist, a language for composition and sound synthesis," Computer Music Journal, vol. 21, no. 3, pp. 50-60, 1997.

[106] M. Laurson, M. Kuuskankare, and V. Norilo, "An overview of PWGL, a visual programming environment for music," Computer Music Journal, vol. 33, no. 1, pp. 19-31, 2009.

[107] M. Rumori, "Sound production and audio programming of the sound installation GROMA," in Proceedings of the 27th AES Convention, New York, NY, USA, 2009, paper no. 7864.

[108] V. Lazzarini, J. Timoney, J. Kleimola, and V. Välimäki, "Five variations on a feedback theme," in Proceedings of the International Conference on Digital Audio Effects (DAFX'09), pp. 139-145, Como, Italy, September 2009.

[109] M. Puckette, "Max at seventeen," Computer Music Journal, vol. 26, no. 4, pp. 31-43, 2002.

[110] Y. Orlarey, D. Fober, and S. Letz, "Faust: an efficient functional approach to DSP programming," in New Computational
Paradigms for Computer Music, G. Assayag and A. Gerzso, Eds., Editions Delatour, Paris, France, 2009.

[111] T. Blechmann, "Supernova-a multiprocessor-aware synthesis server for SuperCollider," in Proceedings of the Linux Audio Conference, pp. 141-146, Utrecht, The Netherlands, 2010.

[112] J. ffitch, "Parallel execution of Csound," in Proceedings of the International Computer Music Conference, Montreal, Canada, 2009.

[113] M. Elizabeth and C. Hull, "Occam-a programming language for multiprocessor systems," Computer Languages, vol. 12, no. 1, pp. 27-37, 1987. 